\title{
Multi-scale Joint Encoding of Local Binary Patterns for Texture and Material Classification
}

\author{
Xianbiao Qi ${ }^{1}$ \\ qixianbiao@gmail.com \\ Yu Qiao ${ }^{2}$ \\ yu.qiao@siat.ac.cn \\ Chun-Guang Li ${ }^{1}$ \\ lichunguang@bupt.edu.cn \\ Jun Guo ${ }^{1}$ \\ guojun@bupt.edu.cn
}

${ }^{1}$ Beijing University of Posts and Telecommunications, P.R. China

${ }^{2}$ Shenzhen Key Lab of CVPR, Shenzhen Institute of Advanced Technology, P.R. China

\begin{abstract}
In the current multi-scale LBP (MS-LBP) on texture and material classification, each scale is encoded into histograms individually. This strategy ignores the correlation between different scales, and loses a lot of discriminative information. In this paper, we propose a novel and effective multi-scale joint encoding of local binary patterns (MSJLBP) for texture and material classification. In MSJ-LBP, the joint encoding strategy can capture the correlation between different scales and hence depict richer local structures. In addition, the proposed MSJ-LBP is computationally simple and rotation invariant. Extensive experiments on four challenging databases (Outex_TC_00012, Brodatz, KTH-TIPS, KTH-TIPS2a) show that the proposed MSJ-LBP significantly outperforms the classical MS-LBP and achieves the state-of-the-art performance.
\end{abstract}

\section{Introduction}

Texture and material classification are a fundamental research problem in computer vision, which play an important role in a lot of vision applications including scene understanding, object recognition, content-based image retrieval, medical image analysis, image segmentation and many more.

The Local Binary Pattern (LBP) [14] descriptor has achieved great success on texture and material classification due to its computational efficiency and texture discriminative power. Since its first publication, LBP has been widely applied to a lot of applications, such as face recognition, face detection, image retrieval, lip reading and many more [17]. A lot of LBP variants have been proposed in the past ten years. In [21], Tan et al. propose a Local Ternary Pattern (LTP) for face recognition. The proposed method shows great robustness to illumination variation. To achieve great rotation invariance, Ahonen et al. [1] propose an effective LBP Histogram Fourier (LBP-HF) features. Their method shows great robustness to image rotation. Recently, a novel Linear Configuration Pattern (LCP) [6] is introduced 

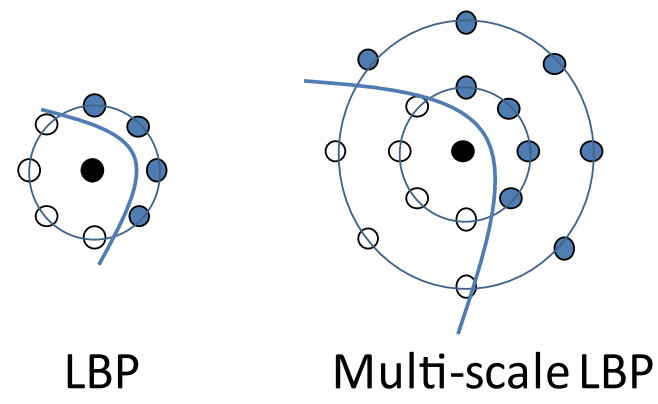

$\begin{array}{ll} & 1 \\ \circ & 0\end{array}$

Figure 1: An illustration of LBP and Multi-scale LBP. Compared with single-scale LBP, LBPs in multiple scales jointly characterize richer local structures. The LBP patterns in different scales have strong correlation.

to explore multi-channel discriminative information of both the microscopic configuration and local features. In addition, there are some other variants, such as DLBP [12], CLBP [8], VLBP [26] and so on.

To depict texture information in different image resolutions, multi-scale strategy is introduced into texture and material classification $[8,9,11,13,16]$. Firstly, single-scale LBP histogram features are extracted in each scale separately. Then, the histograms in each scale are concatenated into a final image representation. Similarly, the same multi-scale strategy are used by LCP, LBP-HF, DLBP and other LBP-based features. Since the multi-scale strategy always achieves much better performance than single scale, it is usually recognized as an indispensable means to achieve the state-of-the-art performance.

However, despite its effectiveness in texture and material classification, the classical multi-scale strategy ignores the correlation information between different scales. As shown on the left panel of Figure 1, each LBP pattern depicts a kind of local image structure. On the right panel of Figure 1, LBP patterns in multiple scales jointly depict a kind of stronger local structure. In fact, texture patterns in different scales around the same central point usually have a strong correlation. Ignoring such correlation will lead to huge lose of discriminative information.

In this paper, we propose a Multi-Scale Joint encoding of LBP ( MSJ-LBP ) feature to encode the joint distribution of LBP patterns in different scales for texture and material classification. Contrast to the classical multi-scale LBP (MS-LBP) that ignores the correlation between different scales, MSJ-LBP can effectively encode this kind of correlation. Compared to the single scale encoding, the multi-scale joint encoding strategy can depict stronger local structures. Meanwhile, the computational cost of MSJ-LBP is extremely low. In practice, the speed of MSJ-LBP is much faster than MS-LBP due to that MSJ-LBP uses a fewer neighbors. In addition, the proposed feature is designed for rotation invariance, and excellent experimental results on the datasets with obvious image rotation demonstrate the robustness of the proposed feature to image rotation. 

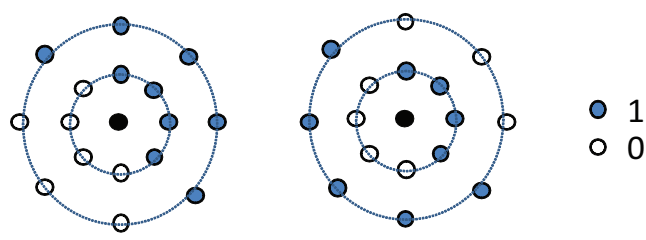

Figure 2: An illustration of drawback of the current MS-LBP. MS-LBP encodes each scale individually. Although the two structures shown above have the same $L B P^{R I U}$ patterns in the corresponding scales, they correspond to different subtle structures in image.

\section{Multi-scale Joint Encoding of Local Binary Pattern}

\subsection{A Brief Review of LBP}

LBP is an effective gray-scale texture operator that depicts local structures of natural images, such as edge, contour, flat region and so on. For each pixel in an image, the LBP pattern can be computed by comparing its pixel value with the values of its neighbors:

$$
\operatorname{LBP}(\vec{s})=\sum_{k=0}^{n-1} \phi\left(v_{k}-v_{c}\right) 2^{k}, \quad \phi(x)= \begin{cases}1, & x \geq 0 \\ 0, & x<0,\end{cases}
$$

where $\vec{s}=[n, r], n$ is the number of neighbors and $r$ is the radius of the neighbors. $v_{c}$ is the gray value of the central pixel, and $v_{k}$ is the pixel value of its $k$-th neighbor.

In [16], Ojala et al. also observed that these patterns with very few spatial transitions described the fundamental properties of the image, and they called these patterns as "uniform patterns". The number of spatial transitions can be calculated as follows:

$$
\Phi(L B P(\vec{s}))=\sum_{k=1}^{n}\left|\phi\left(v_{k}-v_{c}\right)-\phi\left(v_{k-1}-v_{c}\right)\right|,
$$

where $v_{n}$ is set to $v_{0}$. The uniform patterns are defined as those patterns with $\Phi(L B P(s)) \leq 2$. For instance, " 00001110 " is a uniform pattern, while " 00100100 " is not.

The uniform LBP $\left(L B P^{U}\right)$ depends on the start point of the binary sequence. Defined on different start points, $L B P^{U}$ will have different uniform patterns. For instance, the binary sequence " 00001000 " defined on 1 st position or 2 nd position, the corresponding uniform LBP patterns are different. Here, we denote $\operatorname{LBP}^{U}(\vec{s}, i)$ as the uniform LBP pattern on the scale $\overrightarrow{s_{1}}$ with $i$ as the start point of the binary sequence, where $0 \leq i \leq n-1$.

To achieve robustness to image rotation, Ojala et al. also introduced the concept of Rotation Invariant $\operatorname{LBP}\left(L B P^{R I}\right)$ and Rotation Invariant Uniform $\operatorname{LBP}\left(L B P^{R I U}\right)$, where $L B P^{R I U}$ is popularly used for texture classification. The $L B P^{R I U}$ can be defined as:

$$
\operatorname{LBP}^{R I U}(\vec{s})= \begin{cases}\sum_{k=0}^{n-1} \phi\left(v_{k}-v_{c}\right), & \Phi(L B P(s)) \leq 2 \\ n+1, & \text { otherwise, }\end{cases}
$$

For the number of neighbors $n=8$, LBP has $2^{8}=256$ patterns, in which there are 58 uniform patterns and 198 non-uniform patterns. Usually, all 198 non-uniform patterns are summarized into one pattern. Thus, in practice, $L B P^{U}$ has 59 patterns. According to Eq. 3, the $L B P^{R I U}$ has 10 patterns. 

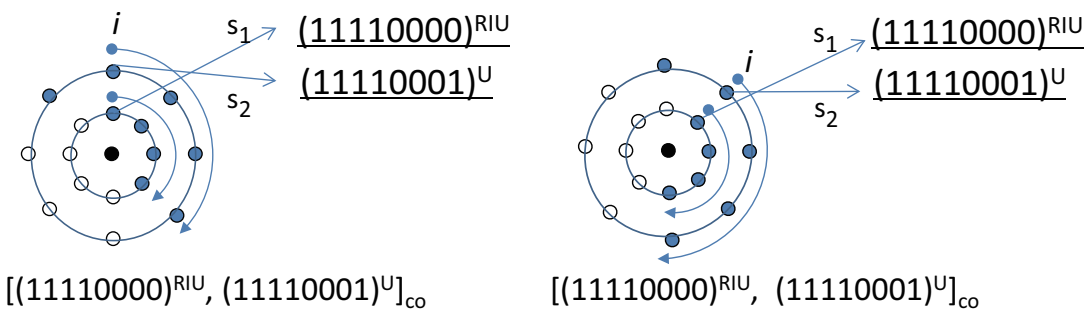

Figure 3: An illustration of the encoding method of MSJ-LBP and its rotation invariance. For the left image, we first compute its $L B P^{R I U}$ pattern in the scale $\overrightarrow{s_{1}}$ and determine the start point $i$ which maximizes the binary sequence. According to the start point $i$, we can compute its $L B P^{U}$ in the scale $\overrightarrow{s_{2}}$. Thus, the joint pattern is $\left[(11110000)^{R I U},(11110001)^{U}\right]$. Similarly, we can get the same joint pattern for the right joint pattern.

\subsection{Multi-scale Joint Encoding Local Binary Pattern}

As shown in Figure 1, each LBP pattern characterizes a local structure, but its descriptive power is limited by its small described region. Compared to single scale LBP, the LBPs in multiple scales jointly characterize richer local structures with larger described region.

To capture the texture information in different resolutions, multi-scale encoding strategy is usually used in texture and material classification tasks $[8,11,13,16]$. First, the $L B P^{R I U}$ histograms are extracted individually from each scale, and then the histograms for all scales are concatenated into the final representation. On most texture and material tasks, the classical MS-LBP achieves great performance .

However, the MS-LBP (Multi-scale $L B P^{R I U}$ ) ignores the correlation between different scales. As shown in Figure 2, the two structures have the same MS-LBP pattern, but they represent apparently different structures. The left figure characterizes a strong contour structure, but the right figure depicts a not usually occurred structure. In the natural images, the LBP patterns between the adjacent scales are usually relevant. The patterns in different scales jointly reflect a stronger structure. Capturing such stronger structure can provide more discriminative information.

To characterize stronger local image structures, it is necessary to jointly encode the joint distribution of LBP patterns in different scales. Here, we propose a kind of multi-scale joint encoding of local binary pattern (MSJ-LBP) feature. Denote MSJ-LBP with scales $\overrightarrow{s_{1}}$ and $\overrightarrow{s_{2}}$ as $\operatorname{MSJ}-\operatorname{LBP}\left(\overrightarrow{s_{1}}, \overrightarrow{s_{2}}\right)$, then it can be defined as follows:

$$
\operatorname{MSJ}-\operatorname{LBP}\left(\overrightarrow{s_{1}}, \overrightarrow{s_{2}}\right)=\left[\operatorname{LBP} R I U\left(\overrightarrow{s_{1}}\right), L B P^{U}\left(\overrightarrow{s_{2}}, i\right)\right]_{c o},
$$

where

$$
i=\arg \max _{i}\left\{R O R\left(\operatorname{LBP}\left(\overrightarrow{s_{1}}\right), i\right) \mid i=0,1, \ldots ., n 1-1\right\},
$$

$\overrightarrow{s_{1}}=\left[n_{1}, r_{1}\right]$ and $\overrightarrow{s_{2}}=\left[n_{2}, r_{2}\right]$. In this paper, $n_{2}$ is set to be $n_{1}$ for efficient computation. $\operatorname{LBP} P^{R I U}\left(\overrightarrow{s_{1}}\right)$ denotes the rotation invariant uniform LBP pattern of the scale $\overrightarrow{s_{1}}$, and $L B P^{U}\left(\overrightarrow{s_{2}}, i\right)$ denotes the uniform LBP pattern of the scale $\overrightarrow{s_{2}}$ using $i$ as the start point. $R O R(x, i)$ performs a circular bit-wise right shift on the P-bit number $x i$ times. $[,]_{c o}$ is a co-occurrence operator.

As shown in Figure 3, we can firstly compute its $L B P^{R I U}$ pattern on scale $\overrightarrow{s_{1}}$ and determine the start point $i$ which maximizes the binary sequence of the scale $\overrightarrow{s_{1}}$. As indicated 
before, $L B P^{R I U}$ has 10 patterns. According to the start point $i$, we can obtain the $L B P^{U}\left(\overrightarrow{s_{2}}, i\right)$ pattern of the center point on the scale $\overrightarrow{s_{2}}$. $L B P^{U}$ has 59 patterns. Thus, the MSJ-LBP includes $10 \times 59=590$ patterns. This encoding strategy is partly inspired by [18]. However, different from [18] and our another work [19] which focus on capturing spatial co-occurrence of two adjacent points on single scale, the proposed method focuses on capturing texture correlation between different scales around one point.

The gradient magnitude reflects the local contrast, and contains a lot of useful information. The regions with large gradient variance usually indicate stronger local edge structures which are informative. Thus, we use the gradient magnitude to weight the joint pattern.

Dense sampling strategy is used for building the histogram like the classical LBP-based histogram representation method. For each point on two chosen scales, we compute its joint MSJ-LBP pattern that is one of 590 patterns and its gradient magnitude. Then, we build the histogram by accumulating the patterns of all points except some boundary points.

\subsection{Image Representation and Classification}

In the previous subsection, we have described the extraction process for two-scales joint patterns. One of these two scales is considered as the reference scale, which is encoded using $L B P^{R I U}$ pattern and provides the start point $i$. Similarly, the other scale can also be considered as the reference scale. Thus, for each pair, we have a histogram with the dimension $590 \times 2=$ 1180. In this paper, we use three scales of $\operatorname{LBPs}(\operatorname{LBP}(8,1), \operatorname{LBP}(8,2)$ and $\operatorname{LBP}(8,3))$ and divide them into three pairs $((8,1)$ and $(8,2),(8,1)$ and $(8,3),(8,2)$ and $(8,3))$. We concatenate the histograms from each pair. Therefore, the final dimension of our feature is $590 * 6=3540$. We can use more scales, but this will further increase the feature dimension but not greatly improve the recognition performance.

To conduct the classification, we use one-vs-the-rest $\chi^{2}$ kernel SVM or nearest neighbor classifier according to the compared previous works. The used $\chi^{2}$ kernel similarity between features $X$ and $Y$ can be written as $S(X, Y)=\sum_{i=1}^{N} \frac{2 X_{i} Y_{i}}{X_{i}+Y_{i}}$, Where $N$ is the dimension of $X$ and $Y$. In practice, we use Vlfeat [24] for efficient computation of kernel matrix. For kernel SVM, we use the Libsvm [5] toolbox.

\subsection{Analysis of MSJ-LBP's Property}

- MSJ-LBP depicts larger supporting region and characterizes stronger local structures. Different from the classical MS-LBP that ignores the texture correlation between different scales, the MSJ-LBP jointly encodes two scales and well preserves the property of the structures.

- MSJ-LBP is gray-scale invariant. The binary comparisons are invariant to the monotonic gray-scale variance. Thus, the MSJ-LBP pattern will not change under the monotonic gray-scale variance.

- MSJ-LBP is rotation invariant. As shown in Figure 3, the proposed encoding strategy promises that MSJ-LBP is invariant to image rotation.

- MSJ-LBP's computational cost is low. In theory, MSJ-LBP has similar computational cost as MS-LBP when they use the same neighbors. But in practice, we use fewer neighbors for the MSJ-LBP to reduce the feature dimension. Therefore, the MSJ-LBP has better efficiency than the MS-LBP. 


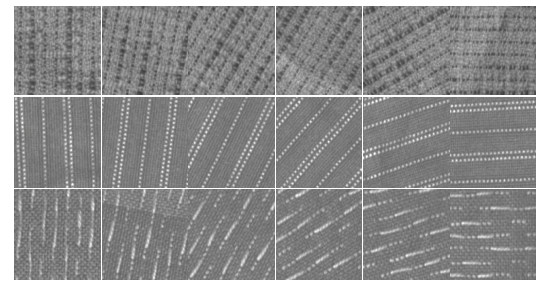

Outex_TC_00012

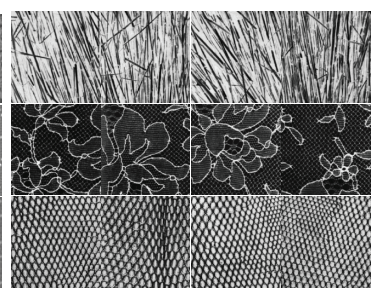

Brodatz

Figure 4: Samples of Outex_TC_00012 and Brodatz. Note that Outex_TC_00012 has strong rotation variation.

\section{Experimental Evaluation}

Baselines. Since the proposed MSJ-LBP is designed to describe the joint distribution of LBPs on multiple scales. Thus, the most relevant competitor is the traditional MS-LBP [16] approach. In texture classification task, rotation invariant uniform $\operatorname{LBP}\left(L B P^{R I U}\right)$ has better robustness to image rotation than the original $\mathrm{LBP}$ and uniform $\operatorname{LBP}\left(L B P^{U}\right)$. Therefore, we use MS- $L B P^{R I U}$ in this paper. Besides of the MS-LBP, there are several LBP variants that show great effectiveness on texture and material classification, such as LBP-HF [1], DLBP [12] and LCP [6]. We will detailly compare our MSJ-LBP with them in the paper.

Implementation Details. In this paper, we just use three scales $(8,1),(8,2)$ and $(8,3)$ for all databases. Thus, we have three pairs $(8,1)$ and $(8,2),(8,1)$ and $(8,3),(8,2)$ and $(8$, $3)$. We also use three scales for MS-LBP and LBP-HF. Their scales are $(8,1),(16,2)$ and $(24,3)$. For LCP, we use the default configuration provided by [6].

Classifier. To fairly compare with previous works, we use the corresponding classifier with them. Since some works use nearest neighbor (NN) classifier, and some previous works use SVM classifier, we use the same classifier as the compared works.

Computational Cost. In theory, the MSJ-LBP has the same computational complexity with the classical multi-scale LBP. However, in practice, our MSJ-LBP is much faster because we use fewer neighbors than MS-LBP. Using the matlab implementation of ${ }^{1}$ on a laptop with Pentium 2.0 GHz Dual-core CPU, MS-LBP takes $116 \mathrm{~ms}$ to process an $200 \times 200$ image, but our MSJ-LBP on matlab takes $55 \mathrm{~ms}$.

\subsection{Evaluation of the effectiveness of joint encoding}

The MS-LBP is the most relevant to our MSJ-LBP. The former individually encodes each scale, but the latter jointly encodes multiple scales. Here, we conduct experiments to directly compare these two approaches to validate the effectiveness of joint encoding strategy.

The experiments are conducted on Brodatz and KTH-TIPS databases. We individually use nearest neighbor and kernel SVM classifiers. For Brodatz, we use 3 samples for training, and the rest for testing. For KTH-TIPS, we use 40 training samples, and the rest for testing. The experimental results are shown in Table 1.

From Table 1, MSJ-LBP significantly outperforms MS-LBP for all configurations. For instance, for the two scale $(8,1)$ and $(8,2)$, MSJ-LBP improves MS-LBP from $87.0 \%$ to $94.0 \%$ using NN classifier. Similarly, we can observe the similar improvement using SVM 
Table 1: Comparison of MS-LBP and MSJ-LBP on Brodatz and KTH-TIPS.

\begin{tabular}{|c|c|c|c|c|c|}
\hline \multirow{2}{*}{ Databases } & \multirow{2}{*}{$(\mathrm{P}, \mathrm{R})$} & \multicolumn{2}{|c|}{ NN } & \multicolumn{2}{c|}{ SVM } \\
\cline { 3 - 6 } & & MS-LBP & MSJ-LBP & MS-LBP & MSJ-LBP \\
\hline \multirow{5}{*}{ Brodatz } & $(8,1)+(8,2)$ & 86.98 & $\mathbf{9 4 . 0 2}$ & 89.46 & $\mathbf{9 6 . 4 6}$ \\
& $(8,1)+(8,3)$ & 87.98 & $\mathbf{9 4 . 7 2}$ & 90.51 & $\mathbf{9 6 . 9 6}$ \\
& $(8,2)+(8,3)$ & 85.36 & $\mathbf{9 3 . 1 7}$ & 87.86 & $\mathbf{9 6 . 2 1}$ \\
& $(8,1)+(8,2)+(8,3)$ & 87.87 & $\mathbf{9 4 . 4 9}$ & 92.03 & $\mathbf{9 7 . 0 5}$ \\
\hline \multirow{3}{*}{ KTH-TIPS } & $(8,1)+(8,2)$ & 91.59 & $\mathbf{9 6 . 1 6}$ & 90.00 & $\mathbf{9 7 . 7 4}$ \\
& $(8,1)+(8,3)$ & 92.59 & $\mathbf{9 6 . 9 3}$ & 89.96 & $\mathbf{9 8 . 0 2}$ \\
& $(8,2)+(8,3)$ & 91.80 & $\mathbf{9 5 . 4 1}$ & 90.79 & $\mathbf{9 7 . 9 8}$ \\
& $(8,1)+(8,2)+(8,3)$ & 93.36 & $\mathbf{9 6 . 8 9}$ & 92.93 & $\mathbf{9 8 . 4 7}$ \\
\hline
\end{tabular}

classifier. According to Table 1 , the joint encoding of scales $(8,1)$ and $(8,3)$ always achieves better performance than $(8,1)$ and $(8,2)$, and $(8,2)$ and $(8,3)$. The reason is that $(8,1)$ and $(8,3)$ has better complementary information than other pairs.

\subsection{Texture Classification and Rotation Invariance}

Outex [15] is a widely used dataset in texture classification. In this paper, we use the challenging test suite Outex_TC_00012. It contains 24 texture classes under different illumination conditions and rotation variance. For each class, 20 samples are used for training. The training images are taken under single orientation, but the 8640 testing images have different orientations. Follow the works $[1,6]$, we use the nearest neighbor classifier. The classification accuracy for different methods are summarized in Table 2.

For all LBP-related methods $\left(L B P^{U}, L B P^{R I U}\right.$, LBP-HF, DLBP, LCP), multi-scale strategy is used. The number of neighbor for LCP and MSJ-LBP are fixed to 8 for all scales, while the number of neighbors for other methods increases with the radius. For example, MSJ-LBP use $(8,1),(8,2)$ and $(8,3)$, but $L B P^{U}$ and $L B P^{R I U}$ use $(8,1),(16,2)$ and $(24,3)$.

Table 2: Comparison of several state-of-the-art methods and MSJ-LBP on Outex_TC_00012.

\begin{tabular}{|c|c|c|c|c|c|c|}
\hline Methods & $L B P^{U}[16]$ & $L B P^{R I U}[16]$ & LBP-HF [1] & DLBP [7] & LCP [6] & MSJ-LBP \\
\hline$R_{1}$ & 56.6 & 64.6 & 77.3 & 56.0 & 68.4 & - \\
$R_{2}$ & 57.8 & 79.1 & 87.3 & 68.7 & 88.1 & - \\
$R_{3}$ & 45.0 & 83.3 & 89.6 & 75.4 & 92.3 & - \\
$R_{1}+R_{2}$ & 59.5 & 82.1 & 89.4 & 77.8 & 84.0 & 91.7 \\
$R_{1}+R_{3}$ & 51.2 & 88.3 & 91.7 & 82.0 & 88.0 & 94.6 \\
$R_{2}+R_{3}$ & 51.3 & 85.7 & 91.5 & 83.7 & 92.7 & 92.7 \\
$R_{1}+R_{2}+R_{3}$ & 53.9 & 87.0 & 92.5 & 84.9 & 90.3 & $\mathbf{9 5 . 1}$ \\
\hline \hline Texton [23] & \multicolumn{7}{|c|}{ MR8 [22] } & \multicolumn{2}{c|}{92.7} \\
\hline
\end{tabular}

From Table 2, MSJ-LBP achieves the highest classification accuracy among all compared methods. The performance of multi-scale uniform $\operatorname{LBP}\left(L B P^{U}\right)$ is $53.9 \%$. It is significantly lower than $L B P^{R I U}$. The reason is that the training samples come from single orientation, but the testing samples are from multiple orientations. $L B P^{U}$ is sensitive to image rotation, but $L B P^{R I U}$ is robust to image rotation. Our MSJ-LBP obtains $95.1 \%$ classification accuracy, which fully verifies the rotation invariance of the proposed method.

Brodatz [2] is a well-known texture benchmark. It contains 111 classes with 9 images per class. The size of each image is $213 \times 213$. We individually use 1 or 3 training samples, and the rest for test. The accuracy is averaged on 100 runs. The results are shown in Table 3. 
Table 3: Performance of kinds of LBP variants and our MSJ-LBP on Brodatz

\begin{tabular}{|c|c|c|c|c|c|}
\hline \multicolumn{7}{|c|}{ Nearest Neighbor Classifier } \\
\hline Methods & MS-LBP & LBP-HF & LBPV & LCP & MSJ-LBP \\
\hline 1 Training Sample & 77.2 & 84.4 & 80.2 & 78.8 & $\mathbf{8 7 . 6}$ \\
\hline 3 Training Samples & 83.2 & 87.2 & 89.0 & 84.8 & $\mathbf{9 4 . 5}$ \\
\hline \multicolumn{7}{|c|}{ SVM Classifier } \\
\hline Methods & MS-LBP & LBP-HF & LBPV & LCP & MSJ-LBP \\
\hline 1 Training Sample & 88.8 & 92.3 & 85.0 & 89.6 & $\mathbf{9 2 . 7}$ \\
\hline 3 Training Samples & 93.5 & 94.6 & 93.2 & 94.4 & $\mathbf{9 7 . 1}$ \\
\hline
\end{tabular}

From Table 3,the MSJ-LBP significantly outperforms the MS-LBP using both NN or SVM classifier. We also observe that the performance of SVM classifier is obviously higher than NN classifier. The observation is consistent with [4, 25]. Meanwhile, the proposed MSJLBP shows better performance than other kinds of LBP variants including LBP-HF, LBPV and LCP. This is mainly due to that MSJ-LBP well considers the correlation between the different scales around one center point, but LBP-HF, LBPV and LCP ignore the correlation and just focus on improving the discriminative power on each scale. It should be noted that our MSJ-LBP is also slightly better than PRI-CoLBP (96.6\%) [18].

\subsection{Material Classification}

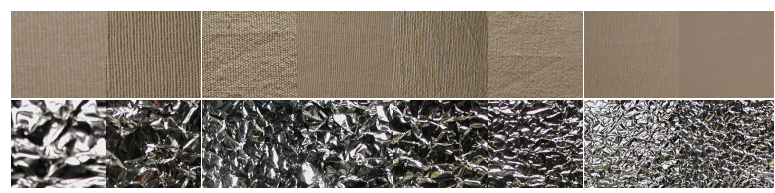

(a) KTH-TIPS

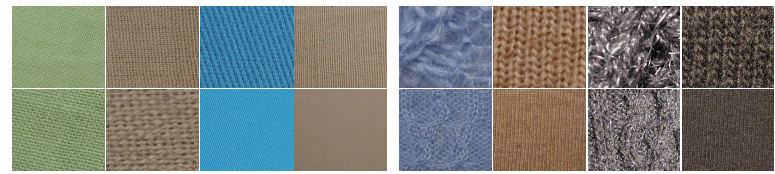

(b) KTH-TIPS2a

Figure 5: Samples of KTH-TIPS and KTH-TIPS2a. Note that KTH-TIPS contains strong scale variation and KTH-TIPS2a has strong scale and appearance variation.

KTH-TIPS [10] database is firstly introduced to bring in scale variation. It contains 10 material classes. The images are captured under 9 different scales (equally spaced from 0.5 to 2), three different illumination directions and three different poses. Thus, in this dataset, each class has 81 samples. Follow the standard experimental setup, we use 40 images per class for training and the rest for testing. The results are shown in Table 4.

From Table 4, we have the following three observations. Firstly, MSJ-LBP achieves the highest performance on all LBP relevant approaches. For instance, using NN, MSJ-LBP achieves 96.5\%, which outperforms LCP for 2.8\%, LBP-HF for 2.2\% and LBPV for $3.5 \%$. Secondly, compared with some state-of-the-art approaches using kernel SVM, such as bag of dense sift (96.1\%) [25], MSJ-LBP achieves better performance. Finally, similar to the results on Brodatz, the performance of using kernel SVM significantly outperforms the NN 
Table 4: Performance of kinds of LBP variants and our MSJ-LBP on KTH-TIPS

\begin{tabular}{|c|c|c|c|c|c|}
\hline \multicolumn{7}{|c|}{ Nearest Neighbor Classifier } \\
\hline Methods & MS-LBP & LBP-HF & LBPV & LCP & MSJ-LBP \\
\hline 40 Training Sample & 93.4 & 94.7 & 93.4 & 94.1 & $\mathbf{9 6 . 9}$ \\
\hline \multicolumn{7}{|c|}{ SVM Classifier } \\
\hline Methods & MS-LBP & LBP-HF & LBPV & LCP & MSJ-LBP \\
\hline 40 Training Sample & 94.4 & 96.9 & 95.5 & 95.9 & $\mathbf{9 8 . 5}$ \\
\hline
\end{tabular}

classifier. With $\chi^{2}$ kernel SVM, our MSJ-LBP gets comparable performance to PRI-CoLBP (98.3\%) [18], but much faster than the latter.

KTH-TIPS2a [3] database includes 4608 images from 11 material categories with 4 different instances per category. All images are captured under 3 varying viewing angles, 4 illumination conditions and 9 different scales. Thus, each instance has $3 \times 4 \times 9=108$ samples. Note that the task is challenging due to that the instances in same categories have huge variation. Follow the setup of [11], we individually use 1, 2 or 3 instances for training and the rest for testing. We also use kernel SVM like [11]. Figure 6 shows the results.

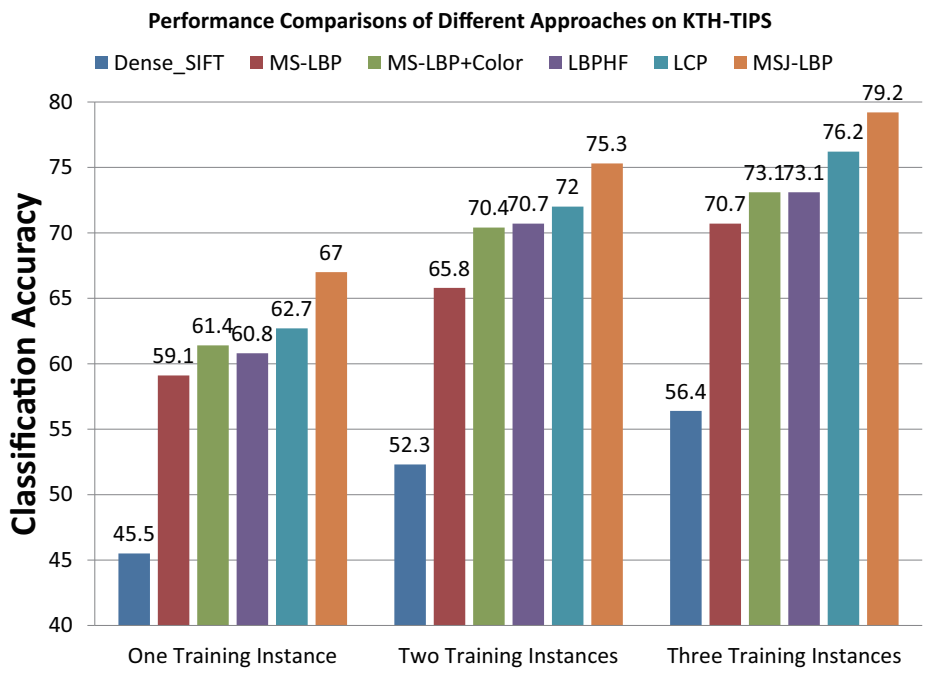

Figure 6: Experimental results of several methods on KTH-TIPS2a.

From Figure 6, it can be observed that MSJ-LBP gets the best performance on all three experimental setups. First of all, all LBP relevant approaches significantly outperforms the bag of dense sift approach. For example, using just one instance for training and the rest three instance for testing, MSJ-LBP outperforms bag of dense sift by about $24.5 \%$. This observation indicates that LBP relevant features may be more suitable to the material recognition task than the SIFT based approach. Meanwhile, although the task is challenging, our MSJ-LBP still achieves obvious improvement than the LCP and LBP-HF. It should be noted that our MSJ-LBP also significantly outperforms newly published LHS (73.0\%) [20]. 


\section{Conclusion}

This paper proposes a novel and effective texture descriptor (MSJ-LBP) for texture and material classification. The proposed MSJ-LBP is computationally simple and invariant to image rotation. Compared to the current multi-scale LBP that ignores the correlation among different scales around the center point, the MSJ-LBP jointly encodes the local binary patterns of two scales around the center point, and can capture the correlation between different scales. We conducted extensive experiments to validate the effectiveness of the proposed approach on texture and material classification task. Superior performances are achieved compared with the state-of-the-art approaches.

\section{Acknowledge}

X. Qi, C.-G. Li and J. Guo are supported by the Natural Science Foundation of China (NSFC) under Grant nos. 61273217, 61175011, and 61005004. Y. Qiao is supported by NSFC (61002042), Shenzhen Basic Research Program, 100 Talents Programme of Chinese Academy of Sciences, and Guangdong Innovative Research Team Program (No.201001D0104648280).

\section{References}

[1] Timo Ahonen, Jiří Matas, Chu He, and Matti Pietikäinen. Rotation invariant image description with local binary pattern histogram fourier features. In Image Analysis. 2009.

[2] Phil Brodatz. Textures: a photographic album for artists and designers, volume 66. Dover New York, 1966.

[3] Barbara Caputo, Eric Hayman, and P Mallikarjuna. Class-specific material categorisation. In $I C C V, 2005$.

[4] Barbara Caputo, Eric Hayman, Mario Fritz, and Jan-Olof Eklundh. Classifying materials in the real world. Image and Vision Computing, 2010.

[5] Chih-Chung Chang and Chih-Jen Lin. Libsvm: a library for support vector machines. ACM Transactions on Intelligent Systems and Technology (TIST), 2011.

[6] Yimo Guo, Guoying Zhao, and Matti Pietikäinen. Texture classification using a linear configuration model based descriptor. In BMVC, 2011.

[7] Yimo Guo, Guoying Zhao, and Matti Pietikäinen. Discriminative features for texture description. Pattern Recognition, 2012.

[8] Zhenhua Guo, Lei Zhang, and David Zhang. A completed modeling of local binary pattern operator for texture classification. TIP, 2010.

[9] Zhenhua Guo, Lei Zhang, and David Zhang. Rotation invariant texture classification using lbp variance (lbpv) with global matching. Pattern Recognition, 2010.

[10] Eric Hayman, Barbara Caputo, Mario Fritz, and Jan-Olof Eklundh. On the significance of real-world conditions for material classification. ECCV, 2004. 
[11] Wenbin Li and Mario Fritz. Recognizing materials from virtual examples. In ECCV. 2012.

[12] Shu Liao, Max WK Law, and Albert CS Chung. Dominant local binary patterns for texture classification. TIP, 2009.

[13] Topi Mäenpää and Matti Pietikäinen. Multi-scale binary patterns for texture analysis. Image Analysis, 2003.

[14] Timo Ojala, Matti Pietikäinen, and David Harwood. A comparative study of texture measures with classification based on featured distributions. Pattern recognition, 1996.

[15] Timo Ojala, Topi Maenpaa, Matti Pietikainen, Jaakko Viertola, Juha Kyllonen, and Sami Huovinen. Outex-new framework for empirical evaluation of texture analysis algorithms. In ICPR, 2002.

[16] Timo Ojala, Matti Pietikainen, and Topi Maenpaa. Multiresolution gray-scale and rotation invariant texture classification with local binary patterns. TPAMI, 2002.

[17] M Pietikäinen, A Hadid, G Zhao, and T Ahonen. Computer vision using local binary patterns. Springer, 2011.

[18] Xianbiao Qi, Rong Xiao, Jun Guo, and Lei Zhang. Pairwise rotation invariant cooccurrence local binary pattern. In ECCV. 2012.

[19] Xianbiao Qi, Yi Lu, Shifeng Chen, Li Chun-Guang, and Jun Guo. Spatial cooccurrence of local intensity order for face recognition. In ICME workshop. 2013.

[20] Gaurav Sharma, Sibt ul Hussain, and Frédéric Jurie. Local higher-order statistics (lhs) for texture categorization and facial analysis. In ECCV. 2012.

[21] Xiaoyang Tan and Bill Triggs. Enhanced local texture feature sets for face recognition under difficult lighting conditions. In Analysis and Modeling of Faces and Gestures. 2007.

[22] Manik Varma and Andrew Zisserman. A statistical approach to texture classification from single images. IJCV, 2005.

[23] Manik Varma and Andrew Zisserman. A statistical approach to material classification using image patch exemplars. TPAMI, 2009.

[24] Andrea Vedaldi and Brian Fulkerson. Vlfeat: An open and portable library of computer vision algorithms. In ACM Multimedia, 2010.

[25] Jianguo Zhang, Marcin Marszałek, Svetlana Lazebnik, and Cordelia Schmid. Local features and kernels for classification of texture and object categories: A comprehensive study. IJCV, 2007.

[26] Lin Zhang, Lei Zhang, Zhenhua Guo, and David Zhang. Monogenic-lbp: a new approach for rotation invariant texture classification. In ICIP, 2010. 